\title{
AUTOMATIZACIÓN EN NIVEL DE CONTROL DE PLANTA MEDIANTE EL USO DE HERRAMIENTAS LIBRES Y COMPUTACIÓN
}

\section{AUTOMATION IN LEVEL CONTROL OF PLANT USING FREE TOOLS AND CLOUD COMPUTING}

\section{RESUMEN}

El control del proceso de fabricación ofrece muchas herramientas para la optimización productiva de una empresa. Existen aplicaciones especializadas para integrar la información a nivel de planta con el de control de empresa, estos software están basados generalmente en sistemas altamente automatizados en los niveles de campo. La industria de muebles para oficina en Colombia está conformada por pequeñas y medianas empresas, con máquinas con un alto contenido de operación humana y baja automatización. El control de producción mediante informes en papel impide que se pueda tener una fotografía en tiempo real del proceso y la gestión puede ser lenta frente a las necesidades de la empresa. En este documento se presenta una alternativa de diseño de un sistema MES basado en herramientas libres y de computación en la nube, además se hace el planteamiento de implementación para la industria de muebles para oficina al ser integrada integrándola con un sistema CAD especializado llamado OffiCAD.

Palabras clave: Automatización, Diseño asistido por computador (CAD), Sistemas de soporte de la manufactura, Sistema de control de ejecución de la manufactura (MES), Google Drive, Muebles para oficina.

\begin{abstract}
The control of the manufacturing process provides many tools for optimizing production company. There are specialized applications to integrate information at the plant level control with company, these software are generally based on highly automated systems at the field level. The office furniture industry in Colombia is made up of small and medium enterprises, with machines with a high content of human operation and low automation. The control of production through paper reports makes it impossible to take a picture in real time and management process can be slow compared to the needs of the company. This paper presents an alternative design of a MES system based on free tools and cloud computing, plus the implementation approach for the office furniture industry is being built by integrating it with a specialized CAD system called OffiCAD.
\end{abstract}

Key words: Automation, Computer Aided Design (CAD), manufacturing support systems, manufacturing execution control system (MES), Google Drive, Office furniture.
Leonardo Emiro Contreras Bravo: Ingeniería Mecánica - Universidad Francisco De Paula Santander - Ufps - Colombia. Maestría en ingeniería materiales y procesos - Universidad Nacional de Colombia - Colombia. Docente - Universidad Distrital Francisco José de Caldas - Colombia - lecontrerasb@udistrital.edu.co

Julián Alfonso Tristancho Ortiz:

Ingeniería Mecánica - Universidad Nacional de Colombia - Colombia. Doctorado En Ingeniería - Universidad De Los Andes - Colombia.

Docente - Universidad Distrital Francisco José de Caldas - Colombia - jatristanchoo@udistrital.edu.co

\section{Luis Fernando Vargas Tamayo:}

Ingeniería Mecánica - Universidad Nacional de Colombia - Colombia. Maestría en Ingeniería Mecánica Universidad Nacional de Colombia Colombia.

Docente - Universidad Distrital Francisco José de Caldas - Caldas - lufvargast@udistrital.edu.co

Tipo: Artículo Artículo Reporte de Caso.

Fecha de Recepción: 26 Abril de 2014 Fecha de Aceptación:24 Junio de 2015 


\section{INTRODUCCIÓN}

El desarrollo de la automatización ha sido un proceso más evolutivo que revolucionario [1]. Cada desarrollo nuevo se ha basado en la evolución de uno anterior debido a la realimentación de los resultados obtenidos.



Figura. 1 Automatización como convergencia de tecnologías [2].

En la actualidad la automatización es una conjunción de varias ramas de la ingeniería, creándose así una ciencia multidisciplinaria como lo explica [2]. En la Figura. 1 se muestran las diferentes ramas de la ingeniería que convergen en la automatización.

Un concepto muy importante que se debe tener en cuenta en la Automatización, es que es un proceso por niveles, formándose una pirámide. En la Figura. 2 se puede apreciar la llamada pirámide de la automatización, donde los niveles más bajos $(1,2,3)$ corresponden a la automatización de máquina, mientras en las etapas posteriores se tienen los temas de automatización organizacional, planeación, económica y administrativa.



Figura. 2 La pirámide de la Automatización [3]

La pirámide de la automatización también muestra el nivel de complejidad e inversión a medida que se asciende. Muestra además un flujo, pero no necesariamente debe ser seguido en orden, la automatización organizacional (control de planta y proceso) puede ser implementada como primera etapa sin intervenir directamente sobre el músculo productivo de la empresa (niveles bajos de la pirámide).

\section{- Objetivos de la automatización}

Todo proceso de automatización tiene una serie de objetivos claros [1]:

1. Integración: La interconexión de las diferentes etapas del proceso productivo, reduce los tiempos que no agregan valor al producto.

2. Mejoramiento productivo: La automatización permite que los costos de producción sean menores.

3. Mejoramiento de la calidad: Los sistemas de control automáticos, permiten que los niveles de precisión sean mucho mejores.

4. Aumentar nivel de seguridad: En procesos que puedan ocasionar problemas de salud o seguridad para los operarios, la automatización es una muy buena alternativa.

5. Reducción de intervención humana: En procesos de alto nivel repetitivo, esto mejora indudablemente la calidad del producto y la salud ocupacional de los trabajadores.

6. Optimización de espacio: La automatización no solo planteada como generación sin 
presencia humana, si no desde el punto de vista organizacional garantiza una optimización del layout y espacio de la planta de producción.

\section{- Relación flexibilidad-productividad}

En la actualidad los cambios tecnológicos, la innovación, la competencia, la globalización y la variación del mercado hacen que los productos (y por ello los procesos de manufactura) cambien rápidamente. Una industria tiene que tener la capacidad de adaptarse a esos cambios o ser flexible [1], pero sin perder significativamente sus niveles de productividad

En la Figura. 3 se puede ver la relación existente entre productividad vs flexibilidad para distintos procesos. A mayor flexibilidad existe un máximo de mano de obra humana, como en el Job Shop. La producción típica de una empresa de este tipo no supera los 100 elementos al año. Su flexibilidad está basada en la capacitación y experiencia de su personal humano.

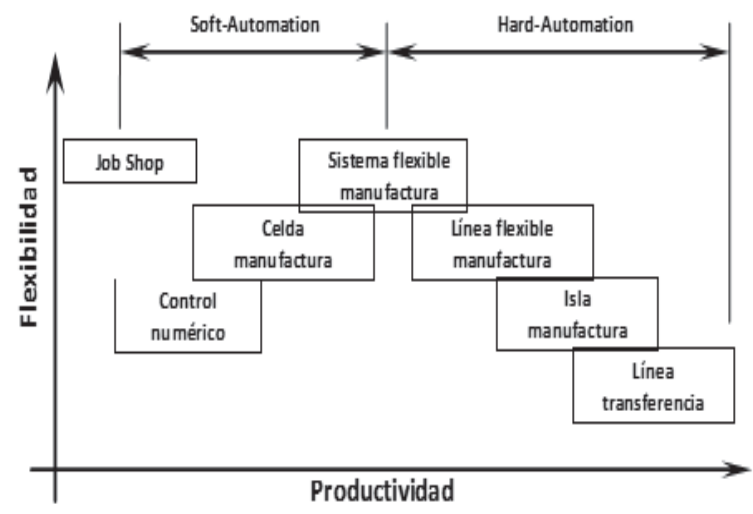

Figura. 3 Flexibilidad y productividad [1].

En la primera etapa de la Figura. 3 (de izquierda a derecha) se tiene la conocida soft-automation, en la cual los sistemas de automatización tienen una muy buena flexibilidad, pero una baja productividad. El termino soft-automation nace debido a que la mayoría de la automatización es realizada por software, por lo tanto un cambio en la producción conlleva un tiempo de programación, pero no un cambio significativo sobre la línea de producción.

Mientras el término hard-automation se acu- ño debido a que la automatización es lograda con hardware o equipos y creación de líneas de manufacturas. Son sistemas con un alto nivel de especialización, cualquier cambio que se desee hacer sobre la línea de producción, implican cambios sobre el layout o equipos ocasionando tiempos importantes de adaptación. De la Figura. 3 se puede concluir que la automatización debe cumplir una condición fundamental, debe estar adaptada a las condiciones y tipo de manufactura de la empresa.

\section{AUTOMATIZACIÓN ORGANIZACIONAL.}

El CIM (Manufactura Integrada por Computador) no se puede decir que simplemente consiste en la conexión de todos los sistemas de hardware y software en una red y luego a un servidor central. Aunque el nombre es engañoso la CIM en realidad es una metodología y una meta más que una simple red [1]. En el mundo existen tres arquitecturas para implementar CIM [2].

1. Modelo Japonés: Basado en la metodología justo a tiempo (JIT). Principal objetivo es eliminar el desperdicio. Donde desperdicio es todo aquello que no le agrega valor al producto. Para ello se utiliza la creación modular de celdas de manufactura usando las técnicas de tecnología de grupos (TG). Todos los elementos deben ser flexibles, es así que los operarios deben tener la capacidad de operar varias o todas las máquinas si llegara a ser necesario.

2. Modelo Estadounidense: En este modelo todos los procesos están centralizados en un solo computador. El objetivo es reducir los sistemas redundantes innecesarios, mejorando las comunicaciones entre los niveles y áreas operativas. Este modelo necesita la automatización de todas las etapas productivas.

3. Modelo Europeo: trata de mezclar los dos sistemas anteriores, con el objetivo claro de sacar las ventajas que tiene cada uno sobre el otro.

Estos tres modelos pese a su alto contenido técnico y de automatización, se dirigen exactamente en la misma dirección, integrar todo el sistema de diseño, mercadeo y producción, 
para lograr hacer de una empresa un ente ágil, rápido e inteligente. Este proceso no necesariamente implica la compra indiscriminada de maquinaria automática, el componente organizacional es aún más importante, que cualquier otro [2].

\section{- Manufacturing Execution Systems (MES)}

Los MES son sistemas informáticos en línea que proporcionan herramientas para llevar a cabo las distintas actividades de la administración de producción, de tal forma que se aumente el nivel productivo de la planta a través de la optimización en la gestión de recursos. Algunos de los objetivos del MES son:

- Medir el rendimiento de las máquinas (OEE: Overall Equipment Efficiency) a través del control del estado de las máquinas.

- La Gestión de Ordenes de fabricación, saber en cada instante, el estado; su cantidad pendiente, realizada, fecha de finalización prevista

- $\quad$ La Trazabilidad ascendente y descendente, incluyendo las condiciones del proceso en la cual se han producido los lotes.

- $\quad$ La logística interna de la planta, actualizando de forma automática los almacenes (producciones y consumos)

- $\quad$ El Control de calidad en planta, manual y automático a través de la integración de equipos de medición.

En un proceso productivo las funciones del sistema MES se realizan mediante la integración de los recursos humanos y sistemas informáticos. En la Figura 4 se puede ver un ejemplo de la estructura de un sistema MES, en cual se tiene la integración de la información por medio de una red corporativa y varios dispositivos electrónicos como PLC, pantallas HMI, computadores de piso y un software que almacena los datos en un base local, desde la cual se puede acceder desde otros clientes para visualizar reportes e información.

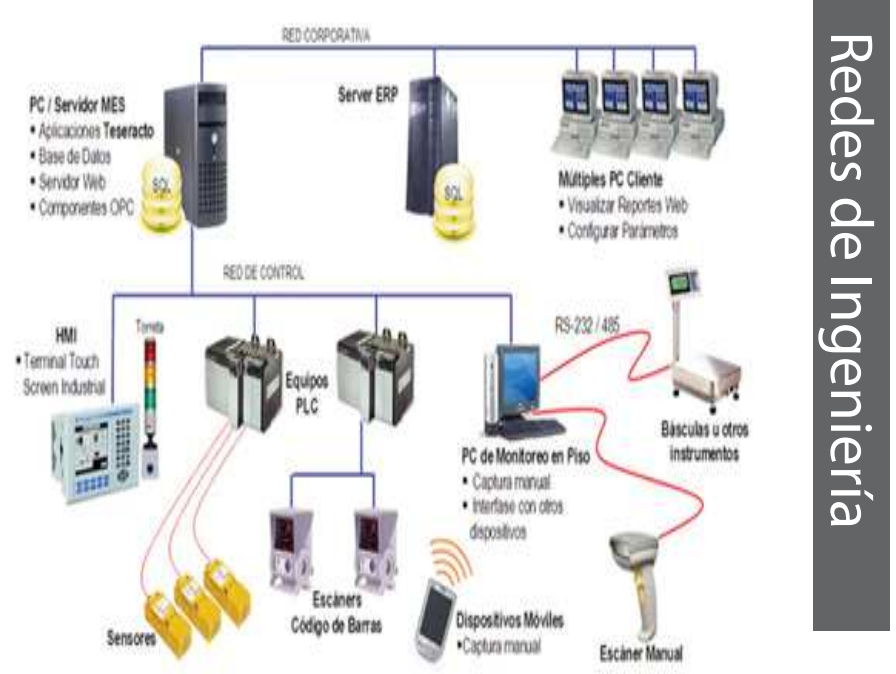

Figura. 4 Arquitectura MES típica y sus componentes (Teseracto, 2014)

El problema fundamental de lograr esta integración son las diferentes aplicaciones e interfaces necesarias para permitir que los sistemas se conecten. Una estructura de este tipo además puede ser muy eficiente en sistemas productivos de gran tamaño, pero en fábricas pequeñas o medianas la inversión inicial es demasiado grande para poder ser implementada.

\section{INTRODUCCIÓN NUEVAS TECNOLOGÍAS DE LA COMUNICACIÓN (TIC) APLICADA A LA MANUFACTURA.}

En la actualidad no solo se ha presentado un incremento importante en las aplicaciones y funciones disponibles en internet para la comunicación empresarial, sino también de hardware de muy bajo costo que puede ser usado tanto por el público en general, como en el ámbito empresarial, gracias a su versatilidad de conexión. A continuación se hace una breve descripción de aplicaciones y hardware que pueden ser usados para implementar un sistema MES.

- Google Drive, Docs y Forms 
Google es una empresa líder en desarrollo de sistemas y servicios informáticos conectados a Internet. Desde su fundación en 1998, se ha fijado como meta la popularización de los servicios de internet, y para ello ha desarrollado una serie aplicaciones de uso libre, entre los cuales se encuentran: suite de programas de oficina (Google Docs), encuestas en líneas (Google Forms) y almacenamiento en la nube (Google Drive). Algunos de estos servicios tienen limitaciones de uso, que generalmente están asociadas al número de visitas o usos diarios permitidos.

Google Forms es una herramienta originalmente diseñada para publicar encuestas en internet, de tal modo que cualquier persona que tenga la dirección URL de la misma la pueda realizar. Los resultados escritos por cada usuario son enviados con un marcador de tiempo (y también identificación única si es necesario) a un hoja de cálculo ubicada en el servicio de almacenamiento en la nube de Google Drive [5]. Al ser un servicio en la nube, desde cualquier dispositivo que soporte conexión a internet, es posible acceder a estos servicios, tanto en modo de visualización como de edición (si posee los privilegios necesarios).

En la Figura. 5 se puede observar los formatos aceptados por Google Drive, donde los Docs son los formatos nativos del sistema, mientras los compatibles son aquellos que se pueden importar y/o visualizar directamente sobre el aplicativo.
Google Docs

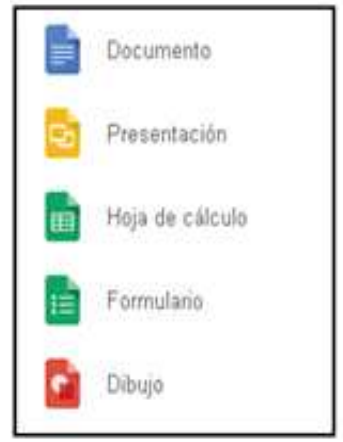

Formatos compatibles

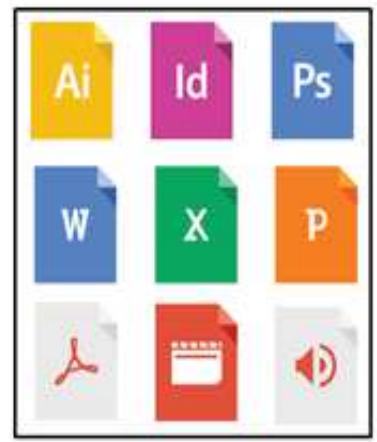

Figura. 5 Formatos de documentos disponibles en Google Drive [5].

\section{- Tecnología inalámbrica de tabletas}

Las tabletas o tablets son computadores portátiles de un tamaño superior a un celular. Sus características principales son: Interconectividad inalámbrica a internet, poder de cálculo mediano, pantalla táctil, diferentes tamaños (habitualmente de 7 a 12 in) y actualmente su bajo costo (pueden llegar a tener un costo inferior a 100 USD).

Estos equipos han tenido un gran impacto en sistemas comerciales debido a su conectividad por internet. Las tabletas y celulares tienen ya un dominio muy marcado sobre: domicilios, redes sociales, juegos y servicios de entretenimiento. En cuanto a su capacidad de computo es aun limitada y pero suficiente para los procesos necesarios de computación en la nube (Cloud Computing).

En la industria ya existen sistemas de soporte de la manufactura que usan las tabletas como sistema principal de visualización y control. Entre las aplicaciones en las que están incursionando se tiene: visualización de tareas y ordenes de producción, sistemas de push to talk (PTT) entre empleados, interfaz hombre máquina móvil de control de procesos, manejo de listas de chequeo de operaciones e inspección de calidad [6].

\section{ESTUDIO DE CASO INDUSTRIA DE MUEBLES PARA OFICINA ABIERTA.}

La historia de la industria del mueble en Colombia ha tenido un fuerte incremento desde mediados de los años 70. La industria del mueble generó ventas en el año 2002 por USD $\$ 163,029,227$, de las cuales USD $\$ 74,863.627$ correspondieron a exportaciones y USD $\$ 39,862.863$ a importaciones, correspondiente al $0.5 \%$ del PIB (Departamento de industria, Comercio y turismo, 2002). Condiciones del mercado como: Recursos naturales disponibles (madera, acero, textiles, etc..), desarrollo de mano de obra especializada y la reactivación del sector de la construcción, garantizan un crecimiento sostenido de este sector [7]. 
El modelo de negocio usado por las empresas de diseño y venta de muebles para oficina abierta se centra en la producción de sistemas modulares e intercambiables que garanticen tener el máximo posible de combinaciones con el mínimo de elementos básicos. Con esta estrategia las empresas pueden generar un inventario muy grande producto, el cual alcanza a tener un tiempo muerto muy grande para salir al mercado. Poder disminuir la cantidad de producto en almacén, reduciendo el tiempo entre el diseño de proyecto e implementación de la entrega y aumento de productividad, son objetivos fundamentales de estas empresas para mantener la competitividad, sobre todo cuando la incursión de producto importado en el mercado nacional es cada vez mayor.

- Generación automática de órdenes de producción

Los proyectos (diseño de espacios de trabajo) que se realizan dentro de esta industria generalmente son personalizados, pues se tienen que acomodar a las condiciones de la empresa contratante, arquitectura disponible y estética [8].

Poder obtener de una manera confiable una cotización rápida para el cliente, que involucre no solo aspectos económicos, sino estéticos y funcionales, se usan generalmente sistemas de diseño CAD (Diseño Asistido por Computador), con los cuales es posible obtener un modelo virtual del proyecto asociado a una lista de partes o BOM (Bill Of Materials).

OffiCAD [8] es una herramienta diseñada en el grupo de investigación DIMSI de la Universidad Distrital, que permite la integración de una base de datos de producto con sistemas CAD de bajo coste, obteniendo como productos finales: cotización para cliente del proyecto, visualización 3D del proyecto y lista de partes para producción. En la Figura. 6 se puede observar la estructura de funcionamiento de OffiCAD.

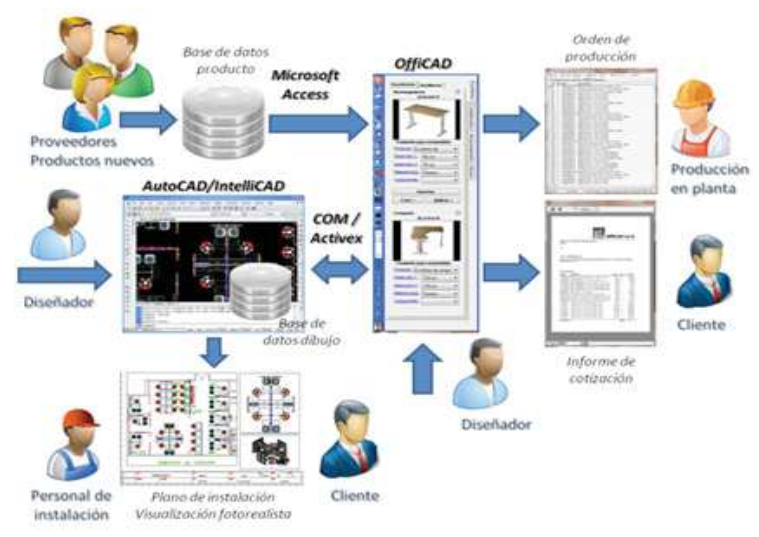

Figura. 6 Estructura de funcionamiento OffiCAD [8].

OffiCAD se puede ubicar parcialmente entre las etapas de control de planta y control de empresa en la pirámide de la automatización, sin cubrirlas en su totalidad. El siguiente paso para aumentar la capacidad de este sistema consistiría en el manejo comercial de la empresa (nivel de control de empresa) convirtiéndolo en un sistema ERP y aumentar su capacidad en el control de planta, pasando de la simple generación de la orden de producción a realizar, a un sistema que cumpla con las tareas básicas de un sistema MES.

La implementación del sistema ERP podría ser incluido con la creación de una base de datos de mayor capacidad tanto de información, como de funcionalidades de reportes y control de clientes, cadena de suministro, inventario y parte comercial.

- Implementación de MES usando computación en la Nube

Para la implementación de un sistema MES acoplado con OffiCAD, se plantea el diseño de un formulario (Google Form) el cual inscribe la información suministrada en una única hoja de cálculo (Google Spreadsheet). En varias hojas de este libro de cálculo se realizan los informes basados en los datos suministrados por el formulario. Para la realización de estos informes se pueden usar técnicas de filtrado y agrupación, con lo cual se puede obtener reportes de: 
- Tiempo de trabajo directo por cada pe dido

- $\quad$ Piezas desechadas o que necesitan tra bajo adicional

- $\quad$ Conteo de piezas terminadas en cada centro de trabajo

- Utilización y tiempo muerto del equipo

- $\quad$ Relojes de tiempo para los empleados

- $\quad$ Tracking sobre el proceso de fabrica ción del proyecto

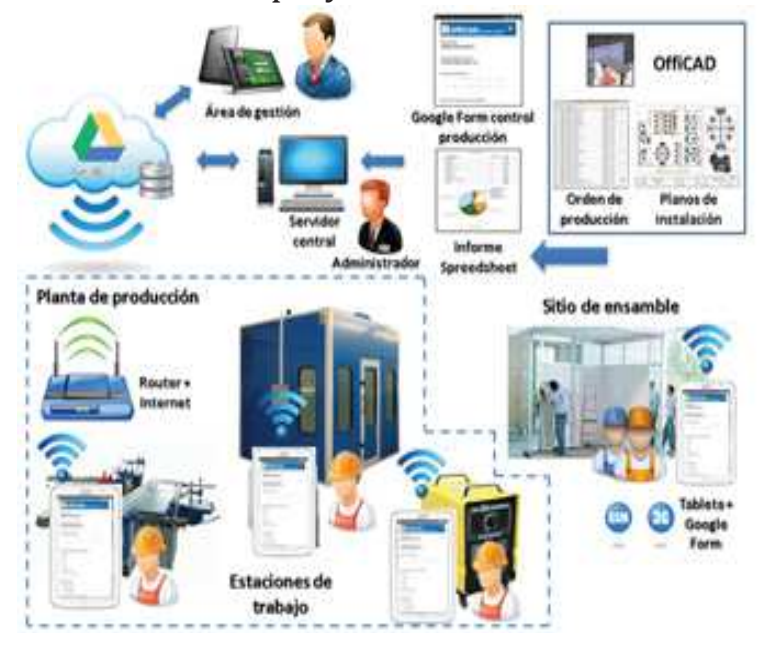

Figura. 7 Estructura de funcionamiento OffiCAD Shop Floor Control

En la Figura. 7 se puede observar la estructura general del sistema. Esta estructura está basada en la arquitectura del modelo estadounidense de Automatización, pues la información se centraliza en un solo equipo que este caso es un servidor en la nube. Cada una de las máquinas o procesos (depende del tamaño de la empresa) posee una tableta (terminal de fábrica), conectada a internet mediante una red WIFI. Esta red es de fácil de implementación y puede cubrir una gran área si se usan los suficientes repetidores o routers.

Cada operario al iniciar turno o labores, mediante el uso de la tableta, un navegador de internet y el formulario generado por el administrador, puede ver las órdenes de producción asignadas por máquina/proceso, ingresando su nombre y seleccionando la información de orden de producción y etapa de fabricación.
Luego a medida que el proceso de fabricación va avanzando, el operario va informado mediante este formulario las paradas necesarias (incluyendo la razón de ellas), al igual que la finalización del proceso y descarga para la siguiente etapa de producción.

El operario a través de la tableta puede recibir información directa de cambio de programación de procesamiento por medio de email o Google talk (Chat de comunicación gratuita), o verificar los procedimientos de fabricación y control de calidad por cada componente. Cada vez que el operario envía información del proceso se escribe con fecha y hora, lo que permite hacer la medición de tiempos de manera completa en el Spreadsheet base.

La administración del sistema se realiza en un servidor central, con el cual se realiza la programación de la producción, se verifica la información enviada y se crean/modifican los informes disponibles. La información usada para el agendamiento y orden de producción del proyecto se obtiene de OffiCAD.

El proyecto al estar en la nube permite que la información sea usada también durante el proceso de montaje final, para ello es necesario el uso de tabletas con acceso a red celular. La información importante para el proceso de montaje incluye los planos, ordenes de producción (agrupada) y renderizados del proyecto.

Los informes de gestión de cada uno de los procesos pueden ser visualizados en tiempo real (no modificados) por el personal de administración, con lo cual se puede hacer correcciones o mejoras del proceso.

\section{CONCLUSIONES}

Este artículo muestra las bases teóricas del control de planta usando herramientas libres y computación de la nube. La viabilidad de implementación de este proyecto está fundamentada en la calidad y confiabilidad de los datos suministrados para la orden de producción. El sistema MES aquí planteado depende de gran 
medida de que los operarios estén comprometidos para la entrega de la información adecuada y verídica en cada uno de los procesos. Esta propuesta de desarrollo es de bajo costo, no necesita desarrollo de software especializado o hardware sobre las máquinas, la única inversión necesaria es la compra de tabletas de bajo coste y entrenamiento de uso a los operarios. La principal desventaja del sistema planteado es la seguridad de los datos, pues al ser un sistema libre la información puede ser fácilmente compartida o espiada por fuera del control de la empresa. Se puede tener control mediante claves y administración de usuarios, pero el nivel de seguridad es muy bajo. También este sistema está planteado para soportar fábricas de tamaño pequeño o mediano, pues el ingreso de información proveniente de muchos puntos haría llegar fácilmente al límite de 400.000 filas, haciendo necesario la fragmentación o cambio repetitivo del Spreadsheet de base. Otra desventaja importante es la dependencia de internet, con lo cual la fábrica tendría problemas muy serios de control si el internet llegase a fallar.

Para mejorar el sistema se podría desarrollar production uptime, Product quality and Profitability", http://www. motorolasolutions.com/. 2010

[7] Gobierno vasco, Departamento de industria, Comercio y turismo. "Programa de Diseño, Producción y Ciclo de Vida, Áreas y Programas Clave Plan de ciencia, tecnología e innovación 2001-2004", Recuperado 8 de Noviembre de 2011 http://www.euskadi.net/pcti/pdf/cas/des areas $/ 2 \% 201$.pdf.

[8] J. Tristancho, L. Vargas \& L. Contreras. "Aplicación del modelo integrado de desarrollo (BIM) en el diseño de proyectos de oficina abierta", Ingeniería, Vol. 16, No. 2, Universidad Distrital Francisco José de Caldas. 2011

\section{Referencias}

[1] S.Kalpakjian \& S. Schmid. "Manufactura, ingeniería y tecnología", 5th Ed, Pearson Eduaction, México. 2008

[2] E. Córdoba. "Manufactura y automatización" Revista Ingeniería e Investigación, Universidad Nacional, Bogotá, Colombia. 2006

[3] M. Groover. "Fundamentals of Modern Manufacturing", 4th Ed, John Wiley \& Sons, United States of America. 2010

[4] Teseracto S.A. "Software de Control de Piso (Shop Floor Control) - Control de Producción", Mexico, Diciembre 2013, http://www.teseracto.com

[5] Google Inc. "Ayuda Google Drive", Recuperado 14 de Diciembre de 2013, https://support.google.com/drive

[6] Motorola Inc. "Innovate to improve 